Special Issue on Longitudinal Methodology, Canadian Studies in Population

Vol. 28(2), 2001, pp. 189-217

\title{
Theoretical and Technical Orientations Toward Longitudinal Research in the Social Sciences
}

\author{
Frans Willekens \\ Population Research Centre \\ University of Groningen \\ The Netherlands
}

\begin{abstract}
:
This paper views life as a developmental process, embedded in a multilevel context. It addresses a number of theoretical, modelling and measurement issues at a relatively abstract level, integrating insights from different disciplines that study developmental processes. At the theoretical level, the usefulness of the life course as an organising principle is discussed. At the analytical level, the main building blocks of the life course are identified, with the life event being the unit of analysis. Each life event has three core aspects: the time at occurrence, the likelihood of occurrence (risk of experiencing the event), and the reason for occurrence. Notions of time, risk, exposure, uncertainty, and interaction are discussed. These basic concepts neatly connect life course theory and life history models.
\end{abstract}

\section{Résumé:}

On considère ici que la vie est un processus de développement, dans un contexte à plusieurs niveaux. Plusieurs questions théoriques, de modélisation, et de mesure sont considérées au niveau abstrait à partir de plusieurs disciplines. Les principaux éléments constitutifs du cours de la vie sont identifiés à partir des événements de la vie. Chaque événement comprend trois aspects: sa place dans l'histoire, le risque qu'il ait lieu, et sa raison d'être. Les questions de temps, de risque, d'exposition, d'incertitude et d'interaction sont considérés. Ces concepts relient la théorie du cours de la vie aux modèles de l'historie de la vie.

Key Words: Developmental process, life course models, biographic theory, methodological individualism. 
Theoretical Issues - Frans Willekens

\section{Introduction}

Populations change because people change, and people change because situations change as new opportunities become available and new restrictions are imposed. This is the basic rationale for a micro-perspective on population dynamics. In the micro-perspective, the way people organise their lives is of major interest. Life is a process that unfolds as the result of the interplay between person and setting. Personal characteristics and contextual factors interact and shape a process of development from birth to death. Some innate factors are universal ultimate goals or preferable end-states; they constitute the major motivations for behaviour. Physical well-being, security, social status, self-esteem and self-actualisation are ultimate goals that motivate behaviour. There are different behavioural routes to achieve the ultimate goals (De Bruijn, 1999 , p. 100). Social rules of conduct, means of existence, and individual choice interact in many ways. The route that is taken is dependent on the socio-cultural and historical context in which people live. In one societal context, social status may require a large family, whereas in another, it may be conditioned on economic independence, which may imply predominance of the professional career and postponement of family formation. Which route is taken and which intermediate goals are set along the way, depend on innate and contextual factors. Nature and nurture are inextricably intertwined.

Life is a process of development along three main dimensions: biology, cognition and emotion. The third dimension is often neglected in studies of life as a developmental process. In demographic research, biological and cognitive processes occupy central positions. As people interact with their environment and exchange information, a mental frame, schema or cognitive structure develops, which represents a coherent and internally consistent view of the world enabling the interpretation of information and of the world as it is experienced. At the same time, however, it constrains the ability to comprehend new and unfamiliar experiences and events. Experiences are always filtered through the current ways of understanding. The organisation of knowledge in cognitive schemes and the adaptation of these schemes to the demands by the environment are two complementary processes underlying cognitive development (Piaget, 1952; quoted by Miller, 1983, p. 71). The first is an internal process and the second relates to the interaction with the environment. Adaptation involves assimilation (fitting information into one's current cognitive scheme) and accommodation (adjustment of the scheme in order to accommodate discrepant information).

The process of development from birth to death in the socio-historical context is the subject of life-course research. Biological and cognitive developments are fundamental processes underlying the order, sequence and timing of many events in the life course. Since biological and cognitive developments proceed 
through stages, and society imposes its own norms in an attempt to regulate the sequence and timing of events, the life course exhibits typical stages. Each stage holds the fruits of the past and the seeds of the future. The transition from one stable stage to the next involves a discontinuity and often involves an unstable and critical period of detachment from the previous period of life to attachment to a new period. This period generally involves a re-assessment of established values, beliefs, guiding principles, behavioural patterns (routine behaviour), etc., and experimentation with new values etc.. The outcome may be substantial reorientations and/or adaptations in life. Transition periods with significant demographic implications include entry into adulthood, entry into empty-nest phase, and retirement from active life. The decisions made during these critical periods often have long-term consequences and involve long-term commitments and irreversibility. Prevailing uncertainties may result in the postponement of critical decisions, especially when the age norms imposed by the social environment are fading. Critical decisions may also be postponed when individuals are not ready yet for the commitments involved or are scared off by the irreversible nature (Birg et al., 1991). The latter may very well explain the postponement of marriage and childbearing that are characteristic of the socalled Second Demographic Transition.

The study of developmental processes requires repeated measurements, longitudinal observation. Longitudinal methodology has been defined in a variety of ways by the disciplines that have used it. Baltes and Nesselroade (1979) describe the history of longitudinal research and provide a rationale. The essence is that agents (or entities) under observation are observed repeatedly as they exist and evolve over time (Baltes and Nesselroade, 1979, p. 4). The aim of longitudinal research is to describe change and to identify the aspects that remain constant during development (constancy amid change; continuity and change). The longitudinal method of observations is closely related to the study of change and development. In demographic and social research, the agents under observation are generally individuals. Change at the macro-level (systems level) is decomposed into changes individuals experience as they age (intraindividual changes) and the impact of situational factors on the change. Several situational factors may be distinguished. They may however be grouped into contemporary factors and historical factors. Of particular interest are historical factors that affect persons in the same stage of life similarly (cohort effects). The individual biographies and the cohort biographies constitute basic concerns in the study of demographic and social change (Ryder, 1965; see also Ulrich Mayer and Huinink, 1990).

Much of demographic research today adopts a life course (life history, biographic) perspective, frequently in combination with a historical (e.g. cohort) perspective. The dominant view is that demographic behaviour is embedded in a broader context of changes at the individual and societal level. The emergence of the life course paradigm is part of important paradigm shifts in the social sciences, including demography. The shift arose out of the confluence of several 
major theoretical and empirical streams of research into human behaviour connecting individual action, social change, and social structure (Elder, 1995; Giele and Elder, 1998, p. 6). The life course perspective offers an opportunity to link behavioural processes in different domains of life. There is need to move beyond the identification of factors that affect demographic behaviour, which is a characteristic of explanatory research, and to pay more attention to how these factors affect behaviour, i.e. the causal mechanism. Many concepts and measurement tools are inadequate to identify causal mechanisms in the lives of people. For instance, the concept of time and the way time is measured (chronological time) are not adequate to unravel developmental processes and to detect important co-evolving processes. Analogously, the concept of risk used in most demographic studies is limited to 'being exposed' or 'not exposed' to an event. The degree of exposure and the factors that affect exposure and hence the risk level (risk factors) do not receive much attention, except in subfields such as family planning and morbidity analysis. The life course perspective invites the researcher to move beyond traditional modes of explanation of demographic change and to aim at uncovering causal processes.

The aim of this paper is to discuss some key issues in longitudinal research. The first issue is the value added by the life course perspective. We intend to show that the life course perspective helps us to understand human behaviour for the simple reason that it focuses on how individuals themselves perceive their lives, not how researchers view reality. Many individuals organise their lives around life events. Demographic events are such life events. Most people spend a considerable part of their life either preparing for a life event or coping with a life event. Some events occur by choice, while other events come by chance. Throughout life, individuals attempt to exert control over the occurrence and timing of these events, to meet higher-order life goals.

The second issue relates to basic concepts that are often used in life course research, but that are frequently defined only loosely. They are time and risk/exposure. For instance, there are several notions of time, chronological time being only one. Exposure is a fuzzy concept that needs to be defined more carefully than in most of the demographic and social science literature. Many of the cases that are included in the 'population at risk' are not at risk or are at reduced risk. Exposure analysis is one of the challenges in life course research.

The third issue addressed in this paper is about the relation between research question, data, and method of analysis. A frequently encountered problem is that data limitations dictate the research questions. Many of the most interesting questions cannot be dealt with because the empirical evidence is lacking. To move beyond the data problem, empirical observations are viewed as manifestations of underlying processes. The emphasis should be on the processes rather than on their manifestations. This has important consequences for modelling. Models should be designed to capture the features of the underlying processes rather than the basic patterns in the data. The types of 
models needed are theoretical models (Burch, 1999). Ideally, the models distinguish between chance and choice, as in real life. That points to the need for probability models (chance) and choice models (e.g. discrete choice).

These three important issues in life course research are addressed in this paper, with the intention of contributing to the discussion that is necessarily incomplete. The main objective is to present the issues in a comprehensive way and to indicate perspectives that resolve associated problems.

The most basic element of the life course is the life event. Although life events and the stages or episodes of life that are marked by the life events are universal, individuals experience the events and go through the stages in infinitely varied ways, that depend on factors that are internal to the individual, on societal factors, and on the situational factors. The structuration of life, i.e., the emergence of a life structure, is different in different contexts. Notwithstanding the universal presence of a life structure, the particular structure that emerges is time-bound and space-bound. The ultimate aim of research is twofold. The first aim is to detect universal properties of events and stages in life, i.e. properties that hold across time and space (in different historical contexts and in different cultures and socio-economic systems). The second aim is to determine how factors that are location-and-period-specific affect people's lives, i.e. how the threads and the historical landscapes (social, economic, cultural, political and technological) are connected. In demography, the properties of interest are (i) the occurrence or non-occurrence of an event in an interval and, in case of multiple events, the number of occurrences, (ii) the timing of the events, and (iii) their sequence. A major interest is in the detection of universal 'life structures' (patterns) and life strategies (behavioural intentions), and of the factors or contingencies (personal and situational) that shape life. The interest in life strategies originates from the awareness that critical decisions in life, i.e. decisions related to life events, are not taken in isolation but are part of general conceptions about future developments in different life domains.

The structure of the paper is as follows. Section 2 positions the emergence of the life course paradigm in demography in a broader context of paradigm shift in social sciences. It demonstrates that the paradigm shift is a fundamental one directed towards the enhancement of our understanding of the causal mechanisms that govern demographic behaviour and population change.

In section 3, life course is characterised as an evolving process of structuration or emergence of a life structure. Experiences and behaviour produce enabling and constraining factors that affect future behaviour. A necessary condition for the study of the structuration of life is that the components of the life structure are defined. The section on the anatomy of the life course presents the universal components of the life course, with the life event being the basic unit. Irrespective of time and space, each life event has three aspects: the time at occurrence, the likelihood of occurrence (risk of experiencing the event), and the 
Theoretical Issues - Frans Willekens

reason for occurrence (effects of other events and processes). These aspects are frequent sources of problems of definition and measurement. The concepts of time, risk, and interdependency are discussed in Section 3.

Section 4 reviews life history models. Models are designed to comprehend reality by capturing the essence. A basic underlying idea is that the life course (life structure) evolves around a series of life events. Each event is the outcome of a process, the event process. Life consists of a great variety of event processes that interact in many different ways and cause the life course to evolve and a life structure to emerge. Important issues in the analysis of life history data are: (i) the identification of the relevant events and event processes, and (ii) the selection of the model that best describes the event process being studied. The choice of model is determined mainly by two factors: the research question and the type of data available. The review of life history models centres around the question of model selection. A distinction is made between two categories of models: probability models that describe event processes and capture the effect of chance, and regression models that capture the influence of explanatory variables on the process being studied. Section 5 concludes the paper. The paper is an adapted version of Willekens (1999).

\section{The Life Course: A Useful Organising Principle}

The emergence of the life course paradigm is part of a broad change in social sciences along four dimensions: (i) from structure to process, (ii) from macro to micro, (iii) from analysis to synthesis, and (iv) from certainty to uncertainty.

\section{From Structure to Process}

When attention focuses on structure of a system, dominant issues are taxonomy or typology and classification, i.e. the ordering of phenomena or items within the structure. Much of life course research is empirical. It concerns the identification and characterisation of stages of life, i.e. the detection and documentation of a structure in the pathways of life. With a growing awareness that behind every entity and structure, there exists a process that creates it, attention turns from structure to process and the emergence of structure (e.g. life structure) as an outcome of interacting processes. Nearly every social and natural science made the transition from entity-oriented perception of reality to process-oriented perception (for details, see Kegan, 1982, p. 13; Blossfeld, 1996).

The same applies to life course studies. Life events are outcomes of processes. Several types of 'event processes' may be considered: random processes, choice processes, social processes, economic processes, etc. The processes point to the causal mechanism underlying an event and to the developmental nature of the mechanisms (also referred to as path dependence). A problem is that events and 
structures can be observed quite easily, but the 'underlying' processes are often difficult to detect. We often see what happens but we rarely know precisely why. To interpret and fully understand events, the underlying, causal processes must be uncovered.

For instance, in many countries we observe that women postpone the first child. We also observe that more women work and that they marry or engage in a stable partnership later. We further observe that women are expected to be economically involved and even economically independent because economic independence is a major aspect of individual autonomy. We finally observe that in countries as The Netherlands, women report (in fertility surveys) that they marry and/or have a child when they are ready for it. The mechanism that leads to the postponement of the first child is not unique.

The complexity of causal mechanisms derives from three important features of developmental processes. First, the ontogenetic nature of processes. Each process has three components: an origin (onset), a progression, i.e. a change in a particular direction, and an end (termination, completion). Ontogeny is the study of the origin, development and direction of a process (see e.g. Baltes, 1997). ${ }^{1}$ Second, the epigenetic nature of processes. In an evolving organism, any process is controlled by the activity of at least one other process. The degree of control depends on the stage of development of the controlling part, according to a more or less stable ground plan (bauplan). Each stage of development creates the basis (developmental readiness) for a transition to a new stage in which other parts of the organism (or domains of life) develop (see e.g. Sugarman, 1986, p. 83). As a result, development is not gradual: stages of rapid development (transition stages) are followed by relatively stable stages. Third, the teleological nature of processes. The processes underlying life events are not entirely beyond the control of the individual. For instance, the birth of a child is an outcome of a choice process as well as a biological process. The control serves a purpose or goal. The degree of control exerted over an event process depends on anticipated effects of the event. The ability of humans to anticipate introduces a major complexity in the event processes.

These three features of a developmental process encompass the three conditions for behavioural change proposed by Coale: 'ready, willing and able.' In a life course context, 'ready' relates to developmental readiness, 'willing' to choice, and 'able' to the ability to exert control over occurrence and timing of life events.

\section{From Macro to Micro}

Coleman (1990, p.2) distinguishes two modes of explanation of the behaviour of social systems (e.g. populations). One studies the behaviour at the system level by identifying system variables that co-vary. A second mode of 
explanation of the behaviour of a system entails examining processes internal to the system, involving its component parts, or units at a level below that of the system. Coleman focuses on the micro-macro link. A population is viewed as composed of agents or actors (individuals, groups, institutions) and population changes are interpreted as outcomes of actions of agents and interactions among agents. Phenomena at the system level emerge from interactions among agents. This mode of explanation is known as methodological individualism. In it, population dynamics is viewed as the composite effect of individual life courses. Another example for the second mode of explanation is multistate demography, in which a population is viewed as being composed of subpopulations. Each subpopulation has its own dynamics and at the same time interacts with other subpopulations. The interactions are manifested in transactions or exchanges, e.g. migration. The behaviour at the population level is viewed as the outcome of the behaviour of the different subpopulations and the exchanges among subpopulations.

The trend from macro to micro is a general one in science and represents the search for elementary building blocks of complex organisms and organizations. In his book Hidden order, Holland (1995) describes the fundamental trend as follows: "If model making, broadly interpreted, encompasses most of scientific activity, then the search for building blocks becomes the technique for advancing the activity.... We gain a significant advantage when we can reduce the building blocks at one level to interactions and combinations of building blocks at a lower level: the laws at the higher level derive from the laws of the lower-level building blocks." (p. 36). The ultimate aim is to comprehend complex phenomena in terms of elementary building blocks, simple rules, or simple principles (see also Burch, 1999, p. 11). Methodological individualism is consistent with that perspective.

A major concern in the shift from macro to micro is the micro-macro link. A feature of any behaviour or process at the micro-level is that it occurs in a particular context. The feature is referred to as embeddedness. The context provides opportunities but imposes restrictions. It enables and restricts individual actions. The context is multilevel and dynamic. It may be viewed as being composed of a large number of processes operating at different levels (layers) of aggregation (e.g. the individual, the family, local neighbourhood, class, ethnic group, state, birth cohort). The processes interact with one another and it is the interaction that shapes development. Although for reasons of analysis, it is often desirable to isolate a process and study its properties in the absence of other processes (in a 'pure state'; in vitro), isolation is never feasible in the real world. Instead of trying to isolate particular events and event processes for study, the focus of attention should be the characterisation of context in terms of multiple processes operating at different levels of aggregation (see e.g. Courgeau \& Lelievre, 1992; Lillard \& Panis, 1998). The study of parallel processes is part of an endeavour to picture demographic behaviour and behavioural patterns during the life course as outcomes of 
interactions between multiple processes (see e.g. Willekens, 1991; Blossfeld et al., 1995)

The view that interaction shapes development and change is receiving a growing attention in demography, not only in micro-level studies, but also in macro-level investigations. For instance, recent studies on the timing and the pace of the demographic transition emphasise the significance of social interactions and the diffusion processes that they generate for the explanation of the onset and pace of fertility transitions (Bongaarts and Watkins, 1996, p. 657).

\section{From Analysis to Synthesis}

Traditionally, demography and other sciences focus on decomposition and analysis. Once the basic entities (components; building blocks) are identified and their position clarified, they may be rearranged to produce different higherlevel population structures. This way, the observations of populations as-weknow-them may be situated within a larger context, i.e. the context of what could be possible. The complement of analysis is synthesis. The synthetic approach constructs aggregates from simple components selected from a finite repertoire and combines them following a system of rules. Rules are abstract, formal specifications. They address the logic of demographic processes and population change. When simple objects or processes are combined according to particular rules, the result may be a complex organism or composite process. Life course, which is a complex and composite process, may be viewed as an outcome of a combination of a large number of elementary rules/processes. The challenge is to detect the elementary processes and the rules that link them. The major tool to link multiple elementary processes to generate complex dynamics and to quantify the contribution of a given process to the complex pattern of change is microsimulation. The use of models to compose complex processes from simple processes has been termed theoretical modelling (Burch, 1999, p. 4) as opposed to empirical modelling which models a specific data set.

\section{From Certainty to Uncertainty}

The occurrences and timing of life events, i.e. the outcomes of event processes, cannot be predicted with certainty. Consequently, events are probabilistic and the event processes are random processes. Probability theory offers the scientific basis for the evaluation of the effect of chance on the human life course. Probability theory associates a probability with each outcome (behaviour) in a range of possible outcomes (behaviours). The result is a probability distribution of the outcomes. For instance, events that occur at a constant rate are more likely to occur sooner than later. That is a feature of the exponential distribution of the time at event, which is directly linked to the constant hazard rate model. In the Cox regression model or the proportional hazard model, which is often used in 
Theoretical Issues - Frans Willekens

demographic research, the effect of the covariates is to postpone the occurrence of the event (covariates reduce the baseline rate of occurrence) or to speed up the occurrence (covariates increase the rate of occurrence).

Uncertainty is an attribute of an event. Following Knight (1964), a distinction is frequently made between risk and uncertainty. A recent study by Schneider $e t$ al. (1998) gives a good overview of conceptual issues surrounding risk, uncertainty and surprise. Risk refers to a condition in which both the event and the probability that it will occur are known. Uncertainty refers to the condition in which the event is known (identified) but its probability of occurrence is not known. Surprise refers to the condition that both the event and the probability are not known. Surprise exists when events cannot be anticipated due to ignorance or denial (unwillingness to recognize that some outcomes are not known). ${ }^{2}$

\section{Anatomy Of The Life Course}

\section{Introduction}

The objective of life history analysis is threefold: description, explanation and prediction. Description involves the detection of a pattern in the timing and sequencing of life events. It determines the particular 'life structure'. The second objective is to determine whether and how different life events are related, i.e. to explain the 'life structure', to identify underlying elementary event processes and to describe how the structure emerges from the interactions among the processes. The third objective is to predict or reconstruct life histories from partial observations. In some fields, e.g. medicine and epidemiology, prognosis is an important dimension of life history analysis. In short, life history analysis is concerned with the description, explanation and prediction of the lives of people, aimed at the explanation and forecasting of population change.

Life history may be described in terms of life events or in terms of states occupied between any two events. The first perspective is dominant in survival analysis and event history analysis; the second in multistate demography. Multistate models relate the state occupied at a given age (and/or time) to the states occupied at previous ages. People are said to have different life histories when differences exist in (i) the types of events that they experience, (ii) the number of events, (iii) the timing of events, and (iv) the sequencing of events. Many factors may explain differences in life histories. Some may be biological, other may be behavioural; some may be concurrent, others may be distal ${ }^{3}$. Some may be associated with individual values and aspirations, other may be related to social norms, cultural beliefs, legal restrictions, and access to economic or other resources (for a discussion, see e.g. Buchmann, 1989, in particular pp. 24ff). A major challenge in life history analysis is to infer the events and processes that shape the life course from the generally incomplete observations. 
Theoretical and Technical Orientations Toward Longitudinal Research

in the Social Sciences

\section{Attributes and Event Types}

The distinction between state occupancy and event is fundamental in life course analysis. At a given age, an individual has a multitude of attributes (personal characteristics, traits): gender, marital status, maternal status [presence/absence of children or number of children], migration status (ever/never migrated), place of residence, living arrangement, health status, employment status, educational status, source of income, etc. The attributes relate to domains of life such as marriage, parenting, and employment. In general, the attributes are objective and measurable. They may also be subjective and refer to values, attitudes, opinions, perceptions, evaluations, etc. (Scott \& Alwin, 1998, pp. 100ff). Some authors view an individual as a 'carrier' of attributes. Most attributes considered in demographic studies have a finite number of categories. Consequently, the variables denoting the attributes are discrete. In modelling, a category of an attribute is referred to as a state. The attribute variable is the state variable. The state occupied or state occupancy refers to the attribute or combination of attributes.

Attributes vary with age. A change in attribute is an event. With each attribute may be associated a career. It represents the changes in the attribute. Examples of careers include the marital career, fertility career, employment career, migration career, housing career, health career, etc. The life history of an individual is fully described if at each age, one knows the attributes of the individual or state occupied. An alternative but equivalent approach to the description of the life course is to record the initial attribute, the time when an attribute changes (i.e. time at event) and the new attribute after the change (direction of change). The first is the status-based approach, which is typical for multistate demography; the second is the event-based approach to the life history recording, which is typical in event history analysis.

\section{Time and Risk}

Time and risk are key concepts in life history analysis. Time raises several substantive and measurement issues. Some are discussed in this section. Most life course studies do not pay much attention to the types of risk and risk levels. Most effort goes to the measurement of the duration at which a person is at risk of experiencing an event. Life history analysis could benefit from a more systematic risk assessment.

\section{a) Time}

It is useful to distinguish chronological time and developmental time. Chronological time is a marker; it locates events on a time scale. Multiple time scales may be used (e.g. calendar time, age, duration of marriage, time since last 
childbirth). A common distinction is between age (individual time), duration of process (process time) and historical time (calendar time). Time scales may be viewed as clocks that start measuring time at different origins. Different measurement scales may be used to express time, as in age-period-cohort analysis. When a continuous measurement scale is used, time is a continuous variable and the associated life history models are known as continuous-time models. When time is measured along a discrete scale (interval or ordinal), time is a discrete variable and the associated life history models are known as discrete-time models (e.g. discrete-time survival analysis).

Chronological time, e.g. age, is not a cause of change or an explanation of biological and psychological maturation. It is a marker of stages in the life course (see e.g. Sugerman, 1986, p. 51; Baltes \& Nesselroade, 1979, p. 5). Developmental time situates events in developmental processes. Referring to child development, Sinclair (1985, pp. 102ff) states that chronological age of a child is the most unreliable guide to assess whether children lag behind in height and weight, since children mature at very different rates. Other age measurements, such as bone age or radiological age (growth of bones, measured radiographically), are more reliable. For instance, the age at which menarche occurs is more closely related to radiological age than to the chronological age. Sometimes, weight is a more important determinant of the onset of a biological developmental process than chronological age. Sinclair discusses several indices of maturity: bone age (radiological age), dental age, sexual age, neural age, mental age, and physiological age. His discussion shows that the chronological age, generally used in life history studies, may need to be reconsidered in favour of developmental time. Developmental time is related to notions such as the stage of development and developmental readiness. The epigenetic principle is central to developmental time.

To complicate matters, there is no single developmental process. Every part of the organism or domain of life has a separate rate of development. Differential growth and development is a main property of human development. Change (growth) in one part may be controlled by the activity of another, and the degree of control or coordination may depend on the stage of development reached by the controlling part. For a given process, the rate of progression does not have to be constant; in most cases it is not constant. The rate is high in so-called transition periods and low in stable periods when few events occur. An example is maturation (biological, psychosocial) and personal growth; these processes may start at different ages, progress at different rates (speed), and may take a lifetime to complete or may terminate at a relatively early age. When related developmental processes do not start at the same time or progress at a different speed, frictions may arise resulting in problems of synchronization ${ }^{4}$ and compatibility. At the individual level, the frictions are associated with the inability to combine different activities or role expectations. For instance, the combination of work (professional career) and family (maternal career) may involve many problems of timing (synchronization) and compatibility. These 
problems are met frequently in studies of simultaneous or parallel careers. Frictions may be removed by rescheduling of activities or events (e.g. postponement of motherhood) or by the introduction of facilities designed to remove incompatibilities (e.g. child care facilities). When an individual maintains different careers simultaneously, some career is dominant at a given moment and the others are subordinate. The dominant career receives most resources, energy, and time (for a discussion, see Willekens, 1991).

At the societal level, many restrictions are imposed on the scheduling of life events to assure an adequate functioning of society. Many social norms and legal restrictions affect the timing and sequencing of life events. Age norms, i.e. social expectations about proper timing of life events, are among the most important. They are in fact indicators of developmental time ('social clock'). A 'social clock' indicates whether we are 'early', 'late' or 'on time' for a significant life event. To be 'off time' frequently invites criticism and social sanction. In some societies, persons may feel to be a failure if they have not found a job by the age of 25 and do not have a child five years after marriage. An age-grade system constitutes a prescriptive timetable for the ordering of life events. Aspects of the age-grade system may be institutionalised, when upper or lower age limits are set for events or activities as legal marriage, school attendance, voting eligibility and retirement (see e.g. Buchmann, 1989). Most age limits are not rigid or strictly enforced.

The measurement of time, even chronological time, may cause several problems. Two problems are singled out.

- The time at which an event occurs is generally different from the onset of the process causing the event. Ideally the time to be considered is the time at onset of the process, although is can generally not be observed. For instance, fertility studies consider the impact of causal factors on the timing (age) of the birth although it would be better to consider the impact on the timing of conception (onset of pregnancy). Mortality studies often consider different causes of death. Most causes are the result of disease processes that constitute the causal mechanisms underlying death. In behavioural studies, measurements of the time at behaviour may lead to erroneous conclusions, since the time elapsed between a decision and actual behaviour may differ for different people (Blossfeld et al. 1995, p.34). In order to comprehend the causal mechanisms leading to events, the focus of attention should be the timing of the onset of processes rather than the timing of the events.

- A particularly difficult problem arises when the measurement scale used by the observer (researcher) differs from the scale used by the subject reporting time (respondent). The distinction relates to the question about the relationship between the objective measurability and the subjective perception of time and the tension between 'external' given time and 
Theoretical Issues - Frans Willekens

'internal' experienced time (see Nowotny, 1994, p. 21). When people are asked about the time or age at which they experienced an important event (e.g. migration, first job, etc.), they may misreport the time or age. Similarly, when asked about the length of an episode (e.g. duration of unemployment, duration of marriage at birth of first child, duration of breastfeeding, duration of current residence, etc.) misreporting may be common, in particular in some societies. Several sources of inaccurate reporting exist, telescoping and heaping being the best known (see e.g. Dex, 1995; Scott \& Alwin, 1998). Heaping refers to the abnormal concentration of responses at certain dates, ages, or durations. Loosely speaking, it may be interpreted as a rounding-off by the respondent at a scale unit coarser than the one formally adopted in the survey, due to 'fuzzy' recall. This problem has important features in common with the time-aggregation bias, which results when the time scale of the (underlying) process being studied does not correspond to the measurement interval (Ter Hofstede \& Wedel, 1998; Cunningham \& Vilasuso, 1995; Petersen, 1993). The rounding-off is related to the perception of time that may vary greatly according to the society under study. The measurement problem has therefore a significant cognitive-anthropological dimension. Heaping is a significant problem in life-history analysis with few solutions offered in the literature. For instance, in most surveys the micro data collected are not adjusted to remove the effects of heaping. Heaping and misreporting of time (age) due to ignorance or recall bias may seriously complicate the analysis and interpretation of life-history data. Misreporting of time may in part be a consequence of the respondent viewing life events not in chronological time but in relation to one another, i.e. in developmental time. For instance, in traditional societies, the time at marriage is expressed relative to menarche rather in terms of age. The misreporting of age or time at event receives little attention in most life history studies.

\section{b) Risk and exposure}

Other key variables in life history analysis are risk and exposure. An individual is exposed or at risk of an event, if he/she can experience the event, i.e. the probability of the event is not zero. For instance, only married persons are at risk of divorce; only fecund women are at risk of conception. The concept of risk is perceived in a variety of ways. In common language, risk refers to the possibility of some damage or failure. In general, risk refers to a positive probability of an event. A person is at risk of an event when the probability of the event is positive. Risk is related to uncertainty. In some fields, e.g. decision theory, a distinction is made between risk and uncertainty. Decisions made under risk pertain to decisions that yield various outcomes with known probabilities. Decisions made under uncertainty refer to a more general case, in which the probabilities of the outcomes are not known (see Fishburn, 1988, p. 78). 
People generally differ in risk level and duration at risk. Indicators of risk level include the probability, the rate, the odds, and the relative risk. Risk indicators are important because of their direct link with life history models: risk indicators are the dependent variables of life history models. The specification of a model implies a choice for a risk indicator. For instance, the probability is the dependent variable in the logistic regression model, while the rate is the dependent variable in the log-rate model and the Cox regression model.

Probability measures the likelihood of an event in a given period. The length of period affects probability. The rate relates the likelihood to the duration at mean risk; it is affected less by the length of period. However, when selection exists, the longer the interval the lower the risk. Ratios of probabilities or rates are used to compare the risk in different populations (e.g relative risk, odds). Since different people have different risk levels, individuals may be assigned to different risk categories. Risk assessment involves the collection of information on individuals and situations to determine the risk level (Williams et al., 1995). The result may be a set of ordered risk categories, e.g. 'low risk', 'intermediate risk', 'high risk,' or it may be a regression model relating the risk level (probability or rate) to characteristics of individuals and situations. Several types of characteristics may be distinguished. For instance, a distinction may be made between risk indicators (e.g. mortality differs by marital status), risk factors (e.g. smoking affects risk of lung cancer), and interventions (e.g. treatment of chronic disease affects the risk of death; contraceptive use affects the risk of conception). The distinction between a risk indicator and a risk factor is considered to be a useful one (Hofman et al., 1996, p. 14). Risk factors are risk indicators with a known causal mechanism. The relative risk, which is a ratio of probabilities, is used often in epidemiology to compare risk levels of two populations that differ in one attribute or a set of attributes.

The measurement of duration of exposure receives much attention in the analysis of life history data, mostly in the context of censoring (see e.g. Yamaguchi, 1991, p. 161). It is useful (i) to view exposure as a stage or episode in the life course, being initiated by the occurrence of an event and terminated by the occurrence of another event, and (ii) to distinguish between the duration of exposure and the duration of observation of persons exposed. In general, it is not feasible to observe people during the entire period they are exposed to the risk of an event under study; often, the observation covers only part (segment) of the period. The observation is said to be censored.

\section{Interdependencies in the Life Course}

Life consists of several domains, such as education, employment, marriage, parenting, and co-residence (or sharing). These domains are role domains. The degree of involvement in a domain may be measured by attainment variables such as years of schooling, occupational status, and number of children. 
Domains are linked dynamically and that dynamic linkage is best captured by the career concept. With each domain of life is associated a career; careers interact with one another and with their common environment. The nature and the extent of the interaction are very difficult to assess in the real world, for several reasons. First, career processes may depend on each other directly or indirectly through a third process. Second, the interaction may involve shifts in timing (e.g. postponement) in order to solve incompatibility problems. In fact, the synchronisation of careers or the coordination of careers and associated activities (temporal strategies) are manifestations of dependencies. Third, the impact of one process on another may become manifest after a long time only. Ideally, the study of the dependence structure involves first the isolation of each process from the influences of concurrent processes, as is done in a laboratory context and in studies of processes 'in a pure state', i.e. undisturbed by the presence of other processes. Once the intrinsic properties of each process are revealed, any set of processes may be combined to find out how they interact. These experiments are not feasible yet, so we are left with the study of interacting processes in situ and cannot do much more than hypothesise about the mechanisms by which career processes interact.

In the life course, roughly two types of dependencies may be distinguished: status dependence and event dependence. In case of a single career, status dependence means that the risk of an event (probability or rate) depends on the state occupied. For instance, the probability of migration depends on the region of residence. Event dependence means that the risk of an event depends on the number of times an event already occurred. For instance, the rate of childbearing is dependent on the number of children already born (parity-specific fertility rates). More complex dependencies may be introduced. For instance, the timing of an event may depend on the state occupied and the time spent in that state. The latter dependency is implemented in the semi-Markov model.

Consider now two careers. Career A is status-dependent on career B if the occurrence of an event in career A depends on the position (stage) occupied in career B. For instance, the probability of having a child in a given year depends on the marital status and employment status. Since the marital status and employment status also vary in time, they are referred to as time-dependent covariates (see e.g. Courgeau \& Lelievre, 1992, Chapters 5 and 6; Blossfeld \& Rohwer, 1995, Chapter 6). Time-dependent covariates may be viewed as parallel careers or as explanatory variables that change in time.

Career A is said to be event-dependent on career B if the occurrence of an event in career A, implying an advancement or progression, is influenced by the occurrence of an event in career B. The event in B may enhance or inhibit the occurrence of an event in A. It may also make the occurrence of an event possible. For instance, most professions require a degree. The onset of the professional career is therefore contingent upon the completion of formal education. An event in one career may trigger an event in another career such 
that the events appear to occur simultaneously. Examples are marriage and migration, retirement and migration, and relocation in relation to a major change in health status.

Most studies focus on status dependence. Three types of time-dependent covariates are distinguished (Kalbleisch \& Prentice, 1980, pp. 122-127; quoted by Petersen, 1995, pp. 469-470):

- Deterministic time-dependent covariates: any function of time that is prespecified. The covariates are also known as defined time-dependent covariates (Blossfeld \& Rohwer, 1995, p. 121).

- Stochastic covariates generated by a stochastic mechanism external to the process being studied (dependent career). Examples are job and wage offers and housing offers and housing prices. This dependence is also known as unilateral dependence or local dependence (Courgeau \& Lelievre, 1992, p. 84) and the covariates as ancillary time-dependent covariates (Blossfeld \& Rohwer, 1995, p. 122). The values of the covariates are not affected by the dependent career (career being studied). Most are variables that reflect changes at the macro-level.

- Stochastic covariates generated by a stochastic mechanism internal to the process being studied ('internal time-dependent covariates'). An example is the dependence of the number of children a couple has on whether they remain married or not. The probability of having another child depends on the marital status and the probability of remaining married depends on the presence of children or intention to have children. Another example is the interaction between living arrangement and health status. For the elderly, there is an effect of health status on living arrangement and a reciprocal effect or feedback of living arrangement on health status. The two phenomena are said to be in reciprocal dependence (Courgeau \& Lelievre, 1992, p. 85). This type of dependence is typical at the individual level for careers in different domains of life and at the level of few individuals interacting with each other (e.g. linked lives; household members). Reciprocal dependence frequently involves selection. Elderly people in different living arrangements differ in health because of selection, not because of the effect of living arrangement on health. The selection is in turn causally connected to the ability to anticipate. Reciprocal dependence raises the issue of reverse causation, i.e. the (direct or indirect) effect of the dependent career on the independent career. Reverse causation is seen as a problem because the effect of a time-dependent covariate on the transition rate is confounded with a feedback effect of the dependent career on the values of the time-dependent covariate. 
Theoretical Issues - Frans Willekens

Blossfeld and Rohwer (1995, pp. 122ff) distinguish two approaches to the investigation of interdependent careers or time-varying covariates. The first, labelled the systems approach, is simply to increase the state space to include all possible values of all time-varying covariates. The drawbacks of this approach are well known in multistate demography. The approach is feasible only when the number of parallel careers is small and when for each career, the number of possible states is limited. Blossfeld and Rohwer refer to the second approach as the causal approach. In this approach, it is assumed that the current rate of change in the dependent career depends on the history of the other careers but is independent of their current state. In other words, when the rate of change is measured for an interval, then the history of the other careers is taken into account up to the current interval. What happens to the other careers during the current interval is not considered. Blossfeld and Rohwer (1995, p. 127) refer to this assumption as the principle of conditional independence for parallel processes. This approach has an important implication for the modelling of event histories. From a technical point of view, there is no need to distinguish between defined, ancillary and internal covariates because these covariate types are treated equally in the estimation procedure. When internal covariates exist, the approach implies the assumption that covariates change their values only at discrete points in time. The approach may be implemented by distinguishing relatively small intervals. The transition rate in the dependent career is then affected by the history of the other careers up to the beginning of the current interval. Another implementation of the approach is to split the original interval (episode) in subintervals (sub-episodes) (and to create a new record of data) each time (at least) one of the covariates changes its value. This approach assures that there is no interval (episode) with more than one transition. The approach is called episode-splitting and is discussed by Blossfeld and Rohwer (1995, pp. 128ff) and Petersen (1995, p. 475). For a recent interesting application of the causal approach to the interaction between marital and fertility careers, see Mills (2000).

\section{Life History Analysis: From Theory to Models}

The life history of an individual is fully described if one knows the attributes of the individual at each age. Since the attributes have a finite number of categories, the variables representing the attributes are discrete or categorical variables. Frequently, the number of categories is limited to two only (e.g. presence/absence of attribute or occurrence/non-occurrence of event). In modelling, the attribute variable is often referred to as a state variable and the category as a state. In Section 3, two approaches to recording the life history were distinguished: the status-based approach and the event-based approach. The distinction is important since it affects the choice of life history model. In general, the status-based approach leads to logit models, whereas the eventbased approach leads to log-rate models. 
Theoretical and Technical Orientations Toward Longitudinal Research

in the Social Sciences

\section{Focus: States Occupied}

There are two ways to denote the state occupied by individual, say $r$. The first is to specify a state variable, say $Y_{r}$. $Y_{r}$ is a discrete variable and its value denotes the state occupied. For instance, in marital status analysis, $Y_{r}$ may take values from one to four for single, married, divorced and widowed. If a person is married, $Y_{r}=2$, and if widowed, $Y_{r}=4$. The second approach is to specify a state vector $\mathbf{Y}_{\mathrm{r}}$. The elements of $\mathbf{Y}_{\mathrm{r}}$ are zero, except for the element that denotes the state occupied. The value of that element is one. The second approach is typical for multistate models. For a sample of $\mathrm{N}$ people, the state vector denotes the number of individuals in each state. That state vector is the basis for models of state occupancies and state transitions. The data that are required are status data.

A vector of state occupancies describes, for a given age or for different ages, the number of subjects or respondents in each state (category). To estimate the parameters of the model, data on state occupancies must be available. Such data are sometimes referred to as status data. They are collected as part of a crosssectional survey or census, a series of cross-sectional surveys, a prospective longitudinal survey (e.g. panel) or a retrospective life history survey. Common examples of status data are:

- current status: status at one point in time

- status at two points in time: current status and status at previous point in time (e.g. 5 years ago; at birth; at age 15)

- status at several points in time (e.g. current status, status 5 years ago, and status at birth).

The state occupied at a given age may be viewed as an outcome of underlying substantive and random processes. For instance, being married at a given age depends on such factors as level of education, economic resources, and social norms. It is also affected by chance. The chance mechanism is pictured by a probability model. The binomial and multinomial distributions are probability models that distribute a given number of respondents among a finite number of states or categories. In case the number of possible states is limited to two, the state variable is a binary variable and the probability model is the binomial distribution. The distribution has one parameter, the proportion of respondents in one of the two states (the first, say). The parameter is denoted by $p$. The binomial distribution is:

$$
\operatorname{Pr}\left\{N_{m}=k\right\}=\frac{m !}{k !(m-k) !} p^{k}(1-p)^{m-k}
$$

where $m$ is the total number of respondents, $k$ the number of respondents with a given attribute (or in a given state), and $p$ the parameter. 
If the proportion in the first state is known, the proportion in the second state is also known since the proportions must add up to one. In case the number of possible states exceeds two, the state variable is a polytomous variable and the probability distribution is the multinomial distribution with parameters $p_{i}$, where $i$ denotes one of the possible states $\left(\sum p_{i}=1\right)$. The total number of respondents is given but the distribution among the states or categories is not known and must be predicted by the model. Note that the binomial distribution may be viewed as a multinomial distribution with two states, with $p_{1}+p_{2}=1$. The multinomial distribution is:

$$
\operatorname{Pr}\left\{N_{1}=k_{1}, N_{2}=k_{2}, \ldots\right\}=\frac{m !}{\prod_{i} k_{i} !} \prod_{i} p_{i}^{k_{i}}
$$

The binomial and multinomial distributions predict the state occupancies at a given age. Several factors may influence the state occupied. To account for the factors, a model is specified that relates the parameters of the binomial and multinomial distributions, i.e. the proportion in a given state, to the explanatory variables. The model is a regression model. The dependent variable is a proportion and the independent variables are the factors affecting the state occupancy at a given age. A simple linear relation between the proportion and the factors may be adequate. To assure that the proportion that is predicted by the model remains within the acceptable range from 0 to 1 , while the factors may range from $-\infty$ to $+\infty$, transformation must be used. In demography, the most common transformation is the logit transformation: the logit of $p$ is a linear function of the explanatory variables. In case of a a binomial random variable, the regression model may be written as:

$$
\eta_{i}=\operatorname{logit} p_{i}=\ln \frac{p_{i}}{1-p_{i}}=\beta_{0}+\beta_{1} x_{1 i}+\beta_{2} x_{2 i}+\ldots
$$

where $\eta_{\mathrm{i}}$ is the logit predicted by the model, $x_{k i}$ is the value of the k-th explanatory variable for individuals in category $i$, and $\beta_{\mathrm{k}}$ a regression coefficient. The logit transformation implies that, as $\eta_{\mathrm{i}}$ changes from $-\infty$ to $+\infty$, $p_{i}$ varies following a (cumulative) logistic distribution. The regression model is known as the logit model and the logistic regression model. Note that the logit transformation implies the following relation between $p_{i}$ and $\eta_{\mathrm{i}}$ :

$$
p_{i}=\frac{1}{1+\exp \left(-\eta_{i}\right)}
$$


which is a logistic function. An alternative is the probit transformation, which implies that as $\eta_{\mathrm{i}}$ changes from $-\infty$ to $+\infty, p_{i}$ varies following a cumulative standard normal distribution.

The logit model or logistic regression is used to predict state occupancies. The dependent variable is a proportion or a probability. A particularly interesting application of the logit model is the prediction of choices people make when confronted with a finite number of alternatives. The state occupied at a given age depends not only on the factors at that age. It depends also on the past, e.g. on the state occupied at a given previous age. For instance, it is likely to observe an individual in the married state, when we know that the individual was married one year ago. The current state occupancy is affected by previous state occupancies. When the status is known at two points in time, the state occupancy at the first point in time should be considered in predicting the current state occupancy. The probability model that predicts the state occupied at a given age from the state occupied at a previous age is the Markov model. The parameter is the transition probability $p_{i j}$, with $i$ the state of origin (state at previous age) and $j$ is the state of destination (state at current age) and

$$
\sum_{j} p_{i j}=1
$$

The Markov model may be derived from the multinomial model (or the binomial model in case of two states) by adding an explanatory variable to denote the state occupied at an earlier point in time. Markov models may be generalized to multistate models by relating the transition probabilities to factors affecting the transition probability from state $i$ to state $j$. Markov models and multistate models are applied to predict changes in state occupancies, i.e. transitions from one state to another.

\section{Focus: Events}

The description of the life course focuses on the states occupied at various ages. An alternative but equivalent description focuses on the life events, i.e. the events that cause a change from one stage (state) of life to another. Of interest are the (exact) timing of the event and the direction of change (i.e. the personal attribute or the state occupied after the event). Data on events may come in different forms, e.g.

- occurrence/non-occurrence of event in observation period (observation window)

- time at event: time may be measured in different time scales (e.g. historical time, individual time [age], exposure time [duration at risk]).

- number of events during observation period. 
Theoretical Issues - Frans Willekens

The occurrence/non-occurrence of an event, the timing of the event and the number of events are viewed as outcomes of underlying substantive and random processes. The occurrences, i.e. the number of people in a sample that experience at least one event during a period, may be described by a binomial distribution, since the total number of people is fixed (see above). The number of times people experience an event during the interval is not restricted to be less or equal to the total number in the population (sample). Some people may experience an event several times (e.g. migration). The Bernoulli model is no longer applicable. Consider an interval of unit length (one year or one month, say) and consider a sample of people stratified based on a given attribute. Let $n_{i}$ denote the observed number of events experienced during a unit interval by individuals in category $i$. The probability of observing a given number of events during an interval follows a Poisson distribution:

$$
\operatorname{Pr}\left(N_{i}=n_{i}\right)=\frac{\lambda_{i}^{n_{i}}}{n_{i} !} \exp \left(-\lambda_{i}\right)
$$

where $N_{i}$ a random variable representing the number of events experienced by all individuals in category $i, n_{i}$ a given number of events (a realization of $N_{i}$ ), and $\lambda_{i}$ the parameter of the Poisson distribution. $N_{i}$ is a Poisson random variable. The parameter $\lambda_{\mathrm{i}}$ is the expected number of events experienced by individuals in category $i$ during a unit interval.

Several factors may influence the number of events during a unit interval. To account for these factors, a model is specified that relates the parameter of the Poisson model to explanatory variables. The model is known as the Poisson regression model. The dependent variable is the expected number of events and the independent variables are the factors affecting the occurrence of events. A simple linear relation between the expected number of events and the factors may be adequate. The number predicted by such a model may be negative, however, which is not acceptable. To assure that the number of events predicted by the model is not negative, a logarithmic transformation is used. The transformation assures that the number of events remains nonnegative when the factors affecting the number range from $-\infty$ to $+\infty$. The model may be written as follows:

$$
\ln \lambda_{i}=\ln E\left[N_{i}\right]=\beta_{0}+\beta_{1} x_{1 i}+\beta_{2} x_{2 i}+\ldots
$$

where $x_{k i}$ is the value of the $k$-th explanatory variable for individuals in category $i$, and $\beta_{\mathrm{k}}$ a regression coefficient. The model is known as the log-linear model. The log-linear model predicts the parameter of a Poisson distribution. The link between log-linear model and Poisson distribution is not always made, although it is a clear link. When a research question calls for the prediction of the number 
of events during an interval, the log-linear model or Poisson regression model is the model to be used.

When the interval is not of unit length, the expected number of events depends on (i) the length of the interval and (ii) the size of the population (sample). More specifically, it depends on the total duration exposed to the risk of experiencing the event, i.e. the person-years or person-months spent by the population during the interval. Let $P_{i}$ denote the time spent in the interval by all individuals in category $i$. The ratio of the number of events $N_{i}$ and $P_{i}$ is the occurrenceexposure rate, i.e. the number of events per year or month of exposure. The rate is denoted by $\mu_{\mathrm{i}}$. When the interval is of unit length, $\mu_{\mathrm{i}}=\lambda_{\mathrm{i}}$. In most applications, the total exposure $P_{i}$ is assumed to be independent of the number of events. It is treated as a fixed number. In that case, the expected rate may be written as

$$
\ln \mu_{i}=\ln E\left[\frac{N_{i}}{P_{i}}\right]=\ln E\left[N_{i}\right]-\ln P_{i}=\beta_{0}+\beta_{1} x_{1 i}+\beta_{2} x_{2 i}+\ldots
$$

The model is known as the hazard model or log-rate model. It was developed independently by Laird and Olivier (1981) and Holford (1980) to analyse mortality rates in the context of survival analysis. The log-rate model is a Poisson regression for rate data. For an introduction, see Yamaguchi (1991, Chapter 4; and Vermunt, 1997). The fixed term $P_{i}$ is sometimes referred to as a weight. The log-rate model and the more general log-linear models with weights imply that the count or frequency $N_{i}$ is Poisson distributed with mean $\lambda_{\mathrm{i}}$, conditional on $P_{i}$. In other words, the Poisson model applies to the ratio (rate) $N$ ${ }_{i} / P_{i}$. The expected number of events is

$$
\ln E\left[N_{i}\right]=z_{i}+\beta_{0}+\beta_{1} x_{1 i}+\beta_{2} x_{2 i}+\ldots
$$

where $z_{i}=\ln P_{i}$ is fixed and often denoted as offset.

\section{Conclusion}

Life is an evolving process consisting of various stages. The understanding of the human life course in all its aspects and in its implications for society is an evolving process too. A milestone in that endeavour has been the emergence of the life course as a new paradigm in many if not most of the behavioural and social sciences. The adoption of a life course perspective signifies a shift from structure to process and a greater attention to micro-level analysis. The significance of the emergence of the life course perspective is considered as important as the introduction of the cohort perspective in the study of demographic change. In fact, the life course perspective and the cohort approach are complementary, a view advocated by Ryder as early as 1965. Ryder 
Theoretical Issues - Frans Willekens

(1985(1965): 31) observes: "The cohort approach to social analysis derives strong support from the continuity of individual life". The continuity is an outcome of early socialisation (early crystallisation of the life course). In recent years, continuity has been replaced by flexibility and individual choice, resulting in greater intra-cohort variation. The cohort remains an important concept in the study of demographic change, but the significance of the life course concept has increased for the simple reason that the sources of flexibility in life, already discussed by Ryder, have increased tremendously.

The life course paradigm raises new challenges. Some of the concepts require a thorough examination. In this paper, two concepts are singled out: time and exposure. Although chronological time is generally used, developmental time may be preferred although at the moment its measurement remains close to impossible. However, to understand human behaviour through the life course, we should understand how individuals perceive and experience life. That includes the perception and experience of time in general, and age and duration in particular. The lack of understanding of the subjective experience of time has consequences beyond the study of time. Part of the inability to unravel the interdependencies among parallel careers in the life course is associated with the fact that people experience time differently and that the duration between a decision and its implementation is not the same for everyone (for a discussion, see Courgeau and Lelievre, 1992, p. 96). Exposure is another concept that deserves close attention. In addition to the duration of exposure, the level of exposure or risk level should be distinguished. Demography could benefit from disciplines, such as occupational epidemiology, where exposure analysis and risk assessment are developed more.

Life as an evolving process may represent an interesting perspective, with great prospects for theory development, but the operationalisation in empirical research and statistical analysis represent a major hurdle. Observations are manifestations or representations of underlying random and substantive processes. Random processes are described by probability models. To capture the effects of chance on behaviour, probability models should be used instead of the more common deterministic models. To account for the effects of personal characteristics and other background variables, the parameters of the probability models are related to the background variables. Different types of models are reviewed in this paper. The choice of model depends on the research question. A study of state occupancies of people at a given age calls for logit models and logistic regression. An interest in state transitions calls for a Markov model or a multistate model. When the research question is about the timing of life events, the log-rate model is to be selected since it is the only model that accounts for the duration of exposure by the individuals at risk of experiencing the event. The models reviewed in this paper are the ones that are commonly used in demographic analysis. They have also a clear link with demographic methods such as the life table. 
The cohort and the life course are important concepts in the study of demographic and social change. They relate to two different levels of analysis. Although cohort membership may be important in some situations, it may be less important in other. The study of demographic change may benefit from a further integration of individual characteristics and cohort characteristics in a multilevel perspective and a multilevel model.

\section{End Notes:}

1. In biology, ontogeny is the name for all the developmental events between the fertilization of the egg to the death of the organism.

2. For instance, the discovery of a hole in the ozone over the South Pole in 1985 came as a surprise because the computer programs written to diagnose the satellite data were specifically instructed to reject measurements that diverged sharply from expected normal conditions. When scientists plotted by hand their own ground-based records, the 'outliers' were not omitted and the increase in ozone was detected (Schneider et al., 1998, pp. 165-166). The example shows that 'knowable remains undetected because of the assumptions or views that frame the question or methods of analysis.' (Schneider et al., 1998, p. 166). In population studies it is not uncommon to reject findings that are not anticipated for various reasons, e.g. they are not consistent with established theories, are not captured by established statistical data collection procedures, or are not socially approved ('deviant' demographic behaviour).

3. For instance, the effect of a causal factor may be apparent only after some time. An infection manifests itself many years later when symptoms indicate the presence of an infection. Hence, when an effect is observed, the causal factor or causal event may be situated long ago. Similarly, intentions underlying behaviour and actual behaviour involve a delay. Lagged or historical causation is an important aspect of life history analysis (for a discussion, see e.g. Baltes \& Nesselroade, 1979, p. 37).

4. Synchronization may be viewed as a problem of matching in time.

\section{References:}

Baltes, P. B. 1997. "On the incomplete architecture of human ontogeny. Selection, optimization, and compensation as foundation of developmental theory," American Psychologist 52: 366-380 
Theoretical Issues - Frans Willekens

Baltes, P. B. and J. R. Nesselroade. 1979. History and rationale of longitudinal research, in J. R. Nesselroade and P.B. Baltes (eds.). Longitudinal Research in the Study of Behaviour and Development. New York: Academic Press. Pages 1-39.

Birg, H., E. J. Floethmann and I. Reiter. 1991. Biographische Theorie der demographischen Reproduktion. Campus Verlag, Frankfurt.

Blossfeld, H. P. 1996. "Macro-sociology, rational choice theory, and time: A theoretical perspective on the empirical analysis of social processes," European Sociological Review 12: 181-206.

Blossfeld, H. P., E. Klijzing and G. Rohwer. 1995. Modelling parallel processes in demography. An application example of the causal approach to interdependent systems. Paper presented at the European Population Conference, Milano, 1995.

Blossfeld, H. P. and G. Rohwer. 1995. Techniques of Event History Modeling. New Approaches to Causal Analysis. New Jersey: Lawrence Erlbaum.

Bongaarts, J. and S. C. Watkins. 1996. "Social interactions and contemporary fertility transitions," Population and Development Review 22: 639-682.

Buchmann, M. 1989. The Script of Life in Modern Society. Entry into Adulthood in a Changing World. Chicago: University of Chicago Press.

Burch, T. K. 1999. Computer modelling of theory: explanation for the $21^{\text {st }}$ century. Discussion Paper no. 99-4, Population Studies Center, University of Western Ontario, London, Ont.

Coleman, J. S. 1990. Foundations of Social Theory. Cambridge: The Belknap Press of Harvard University Press.

Courgeau, D. and E. Lelievre. 1996. "Changement du paradigm en demographie [Shifting the paradigm in demography]", Population 51: 645-654.

Courgeau, D. and E. Lelievre. 1992. Event History Analysis in Demography. Oxford: Clarendon Press.

Cunningham, S. and J. Vilasuso. 1995. "Time aggregation and causality tests: results from a Monte Carlo experiment," Applied Econometrics Letters 2: 403-405.

Dex, S. 1995. "The reliability of recall data: a literature review," Bulletin de Methodologie Sociologique 49: 58-89. 
Theoretical and Technical Orientations Toward Longitudinal Research

in the Social Sciences

De Bruijn, B. 1999. Foundations of Demographic Theory: Choice, Process, Context. Amsterdam: Thela-Thesis Publishers.

Elder, G. H. Jr. 1995. The life course paradigm: social change and individual development, in: P. Moen, G.H. Elder Jr. and K. Luscher (eds.) Examining Lives in Context: Perspectives on the Ecology of Human Development. Washington D.C.: American Psychological Association. Pp.101-139.

Fishburn, P. C. 1988. Normative theories of decision making under risk and under uncertainty. Pp.78-98 in: D.E. Bell, H. Raiffa and A. Tversky (eds.) Decision Making. Descriptive, Normative, and Prescriptive Interactions. Cambridge: Cambridge University Press.

Giele, J. Z. and G. H. Elder Jr. (eds.) 1998. Methods of Life Course Research. Qualitative and Quantitative Approaches. Thousand Oaks: Sage Publications, Ca.

Hofman, A., D. E. Grobbee and J. Lubsen. 1996 Klinische Epidemiologie (Clinical Epidemiology). Utrecht: Wetenshappelijke Uitgeverij Bunge.

Holford, T. R. 1980. "The analysis of rates and survivorship using log-linear models," Biometrics 65: 159-165.

Holland, J. H. 1995. Hidden Order. How Adaptation Builds Complexity. Reading, Mass: Addison-Wesley.

Kalbfleisch, J. and R. Prentice. 1980. The Statistical Analysis of Failure Time Data. New York: Wiley.

Kegan, R. 1982. The Evolving Self. Problem and Process in Human Development. Cambridge: Harvard University Press.

Knight, F. H. 1964. Risk, Uncertainty and Profit. New York: Century Press. (originally published in 1921).

Laird, N. and D. Olivier. 1981. "Covariance analysis of censored survival data using log-linear analysis techniques," Journal of the American Statistical Association 76: 231-240.

Lillard, J. A. and C. W. A. Panis. 1998. Multiprocess multilevel modeling. Paper presented at the Annual Meeting of the Population Association of America, Chicago, 1998.

Miller, P. H. 1983. Theories of Developmental Psychology. San Francisco: W.H. Freeman and Co. 
Theoretical Issues - Frans Willekens

Mills, M. 2000. The Transformation of Partnerships. Canada, the Netherlands and the Russian Federation in the Age of Modernity. Amsterdam: Thela Thesis.

Nowotny, H. 1994. Time. The Modern and Postmodern Experience. Cambridge: Polity Press.

Petersen, R. 1993. "Recent advances in longitudinal methodology," Annual Review of Sociology 19: 425-454.

Petersen, T. 1995. Analysis of event histories. in: G. Arminger, C. C. Clogg and M. E. Sobel (eds.) Handbook of Statistical Modeling for the Social and Behavioral Sciences. New York: Plenum Press. Pp.453-517.

Piaget, J. 1952. The Origins of Intelligence in Children. New York: International University Press.

Ryder, N. 1965. "The cohort as a concept in the study of social change," American Sociological Review 30: 843-861. Reprinted in: W. M. Mason and S. E. Fienberg (eds.). 1985. Cohort Analysis in Social Research. Pp. 9-44. New York: Springer Verlag.

Schneider, S., B. L. Turner II and H. Morehouse Garriga. 1998. "Imaginable surprise in global change science," Journal of Risk Research 1: 165-185.

Scott, J. and D. Alwin. 1998. Retrospective versus prospective measurement of life histories in longitudinal research, in: J. Z. Giele and G. J. Elder Jr. (eds.) Methods of life course research. Qualitative and quantitative approaches. Thousand Oaks, Ca: Sage Publications. Pp. 98-127.

Sinclair, D. 1985. Human Growth After Birth. 4th Edition. Oxford: Oxford University Press.

Sugarman, L. 1986. Life-span Development. Concepts, Theories and Interventions. London: Methuen.

Ter Hofstede, F. and M. Wedel. 1998. "A Monte Carlo study of time aggregation in continuous-time and discrete-time parametric hazard models," Economics Letters 58: 149-156.

Ulrich Mayer, K. and J. Huinink. 1990. Age, period, and cohort in the study of the life course: a comparison of classical A-P-C analysis with event history analysis or farewell to Lexis? in D. Magnussen and L. Bergman (eds.) Data Quality in Longitudinal Research. Cambridge: Cambridge University Press. Pp. 211-232. 
Theoretical and Technical Orientations Toward Longitudinal Research

in the Social Sciences

Vermunt, J. 1997. Log-linear Analysis of Event Histories. Thousand Oaks, Ca.: Sage Publications.

Willekens, F. J. 1991. Understanding parallel processes, in: J. J. Siegers, J. de Jong-Gierveld and E. van Imhof (eds.). Female Labour Market Behaviour and Fertility: Preferences, Restrictions and Behaviour. Berlin: Springer Verlag. Pp.11-31.

Willekens, F. J. 1999. The life course: models and analysis, in: L. J. G. van Wissen and P. A. Dykstra (eds.) Population Issues: An Interdisciplinary Focus. New York: Kluwer Academic/Plenum Publishers. Pp.23-51.

Williams, C. A. Jr., M. L. Smith and P. C. Young. 1995. Risk Management and Insurance. Seventh Edition. New York: McGraw-Hill.

Yamaguchi, K. 1991. Event History Analysis. Newbury Park, Ca.: Sage Publications. 